\title{
Tangential surfaces to optimize digital manufacturing of complex shapes
} al Conference of the Iberoamerican Society of Digital Graphics Medellin | Colombia

\author{
Emanuela Lanzara \\ University Federico II | Italy | emanuela.lanzara@unina.it \\ Mara Capone \\ University Federico II | Italy | mara.capone@unina.it
}

\section{INTRODUCTION}

Computational Geometry research field is aimed at complex systems and data management according to new potential of digital tools for advanced shaping, new manufacturing processes and ecological strategies. Advanced design digital approaches require to put together theoretical notions and sociological dimensions. One main goal is to introduce new sustainable methods and technologies to meet ecologic and economic challenges. This purpose needs to develop a digital conscience to create future competent figures and to improve professionals activities in design field. From research to teaching, numerous current best practices confirm Academy as entrepreneurial incubator. It is important above all to conceive craftsmanship and product engineering aimed at promoting the use of recyclable materials and resources optimization. Progress achieved in these fields promotes experimental use of new materials but also innovative use of traditional materials, such as wood or cardboard (Menges, 2011). Therefore, this paper is aimed to explicit link between techniques, materials and geometry to achieve functional, ecological, economic and aesthetic approaches aimed at design and manufacture optimized products.

\section{METHODOLOGY}

In Architecture and Design fields, basic and advanced geometric-mathematical rules allow to solve several problems about design and production of complex systems. Current evolution of digital manufacturing technologies and digital generative algorithmic design tools influence designer thinking and results. Combination of geometry and current digital manufacturing techniques allows complex configurations unthinkable before using traditional materials. Digital modeling of prototypes showed and described in following paragraphs is based on an algorithmic - generative visual scripting approach (Rhino3D and Grasshopper $\mathrm{McNeel}$ ) and geometric and mathematical principles to manage primitive or complex shapes and patterns. The main goal is to optimize their digital manufacturing testing and combining alternative manufacturing techniques. This paper shows some experimental prototypes resulting by merging different digital fabrication techniques, such as 3D printing and Kerfbending, to manufacture complex shapes starting from the same theoretical approach: transformation of nondevelopable complex surfaces (double curvature) in developable tangential surfaces (single curvature).

\section{THEORY: DEVELOPABLE SURFACES}

Differential classification of surfaces introduced by Leonhard Euler (1707-1783), and subsequently used by Monge, allows us to group surfaces according to the definition of curvature, which will be precisely defined by Carl Friedrich Gauss in 1902 (Gauss 1902), in four categories: surfaces with zero curvature $(k=0)$, surfaces with positive curvature $(k>0)$, surfaces with negative curvature $(k<0)$ and surfaces with variable curvature.

Surfaces with zero curvature are specific ruled surfaces, also called developable surfaces.

From a theoretical point of view, we know that:

- all developable surfaces are ruled surfaces;

- all developable surfaces are surface with zero Gaussian curvature; 
- developable surfaces can be generated by tangent line motion on a space curve;

- a surface is developable if two consecutive generating lines cross each other.

According to these premises, developable surfaces can be grouped in conical surfaces, cylindrical surfaces and tangential surfaces (Figure 1).

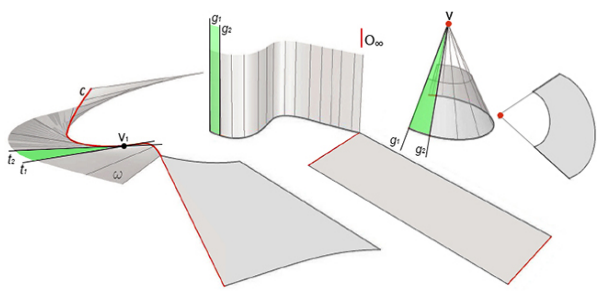

Figure 1: classifications of developable surfaces.

The intersection point between two consecutive generating lines can be proper $(\mathrm{V})$ or improper $\left(\mathrm{O}_{\infty}\right)$

Generating a conical or a cylindrical surface is easy: assigned a directrix and a projection center (proper or improper point) it is possible to construct $n$ generating lines joining the center and $n$ points of the directrix. It is possible to easily generate these surfaces using a 3D modeling software to extrude a curve along a specific direction or to a point. It's much more complex to generate a developable tangential surface: it is very easy to find conical or cylindrical surfaces development using traditional methods or digital tools; on the contrary, it is more difficult to find the unrolled shape of a developable tangential surface (Capone \& Lanzara, 2018). This research activity focuses on design and manufacturing of tangential surfaces.

\section{DEVELOPABLE TANGENTIAL GENESIS}

In Descriptive Geometry a tangential developable is generated from a spatial curve as the surface built on its consecutive tangent lines. This group of ruled surfaces can be generated using only one directrix (edge of regression) because the tangent in each point is unique and it is always uniquely determined (Migliari, 2009, p. 160). The advances in applied geometry derives from use of digital tools that allow to automatically construct a tangent line at a point in a spatial curve. To generate the surface, first it is necessary to construct $n$ tangents which, although automatically, must be determined one by one. Then, the surface can be generated considering these $n$ generating lines. The difference between the generated surface and the tangential development depends on the number of generating lines. Gaussian curvature analysis tool (colorful gradient) allows to verify if the achieved surface is developable (green: $k=0$ ) or not (blue: $k<0$ ).

A developable tangential can be unrolled with some unavoidable approximations, as the two consecutive generating lines intersect each other's on the edge of regression only in an infinitesimal neighborhood, with $n$ tending to infinity. As part of experimentations, we have done a definition that follows geometrical principles to construct developable ruled surfaces starting from a generic spatial curve. Dividing the assigned spatial curve (the edge of regression) in $n$ parts, our algorithmic definition allows to generate the surface by extracting $n$ tangent lines (generating lines) on $n$ points of the curve and tangent to it. By modifying the length of the generating lines and the shape of the edge of regression it is possible to achieve infinite developable configurations (Farinella \&Baglioni, 2017).
Finally, these surfaces may be cut to define the external edge which is otherwise automatically generated as a function of the generatrix length (Figure 2).

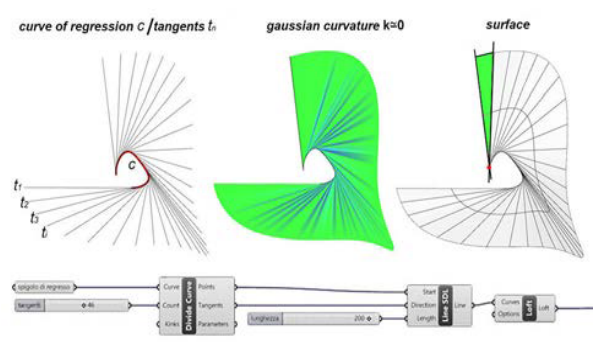

Figure 2: algorithmic-generative definition of a developable surface built on a set of tangent lines. Increasing the number (variable) of generating lines the surface approximation improves (all zero curvature).

\section{UNROLLED SHAPES FROM DEVELOPABLE TANGENTIALS}

A surface, as we said, is developable if it can be unrolled on a plane with rigid movements (isometric transformation), without stretching or tearing. This condition is possible only if two consecutive generating lines are coplanar.

According to our goals, we have analyzed principles and methods used to find unrolled shape of tangential developable. We have defined different approaches that allow us to determine the approximate unrolled shape of a non-developable surface. We have also considered materials and manufacturing techniques that can solve approximation problems connected to smash, allowing the panel deformation thanks to cuts, kerfing, or overlapping, bending.

As we noted, developable surfaces have zero Gaussian curvature, consequently they can be manufactured, using a flexible and non-deformable material, starting from their unrolled shape simply by shaping the cut-out shape. This kind of surfaces are easy to manufacture and this property has favored their diffusion (Lawrence, 2011).

In the case of cylindrical surfaces, all the generating lines are all incidents in one point at infinity. Using the traditional method introduced by Monge, to determine the development of the surface, the directrix is divided into $n$ parts and a prismatic surface is obtained. Cylindrical surface is developed by unrolling the $n$ quadrilateral faces in sequence on a plane. Furthermore, conical surfaces can be considered developable surfaces because two consecutive generating lines are always intersecting lines. To define the unrolled surface, we divide the directrix into $n$ parts and we transform the continuous surface into a discrete surface: a pyramid. Using 3D modeling software, we can automatically unroll both conical surfaces and cylindrical surfaces. Finding the unrolled shape of a tangential developable is more complex: the applications of Differential Geometry principles is evident. According to differential calculus, Monge studies the properties of developable surfaces (Migliari, pp. 106 - 108). Each developable surface can be flattened onto a plane without distortion and, in a limited region, without overlapping. The unrolled shape of the surface generated by the infinite tangents to a space curve is obtained by considering $n$ generating lines and flattening onto plane the surfaces included between two consecutive generating lines (Figure 1). 
If we consider two consecutive tangents $t_{1} t_{2}$, in theory incidents, they identify a plane. By rotating $t_{2} t_{3}$ around $t_{2}$ and repeating the operation for the subsequent tangents we find the unrolled surface. The unrolled surface depends on the edge of regression. It may happen that the configuration of the surface is such that portions of unrolled surface overlap with the others (Fallavollita, 2008), in these cases, it is necessary to divide the design surface into parts in order to manufacturing it.

According to these theoretical premises, we have defined an algorithmic tool that allows to find the unrolled shape of any tangential surface. We know that if we divide a space curve (edge of regression) into $n$ parts and we consider $n$ tangents (generating lines of ruled surface), two consecutive tangents intersect each other along the edge of regression. This is true only in a small, infinitesimal neighborhood of each point. In fact, if we divide the edge of regression into $n$ parts and we consider two consecutive tangents, $t_{1}$ and $t_{2}$, led respectively by points 1 and 2 , we define the non-flat quadrilateral A12B. When point 2 goes to point 1 , then points 1,2 and $B$ can be considered aligned and, approximately, it will always be possible to define a flat triangular face and then to unroll the polyhedral strip composed by $n$ triangular faces. The approximation of the unrolled surface obviously depends on $n$.

We have done two experiments and we have analyzed the results to evaluate which method allows to achieve the unrolled shape that best approximates and fit the real surface. In the first case we have divided the surface using $n$ tangents and we have defined a sequence of surfaces modeled by using no - flat trapezoid A12B. Therefore, as we have previously said, they cannot be unrolled, and they have been flattened using smash digital tool which allows us to determine the approximate development of a nondevelopable surface. We have determined the unrolled shape of the surface by sequentially unrolling the quadrilaterals onto plane. In the second case, we have supposed that the points 1,2, B, and 2, 3, C are aligned and we have split the surface into triangles $A B 1, B C 2, C D 3$, and so on, and we have determined the unrolled shape by unrolling all the consecutive triangles onto plane (Figure 3 ).

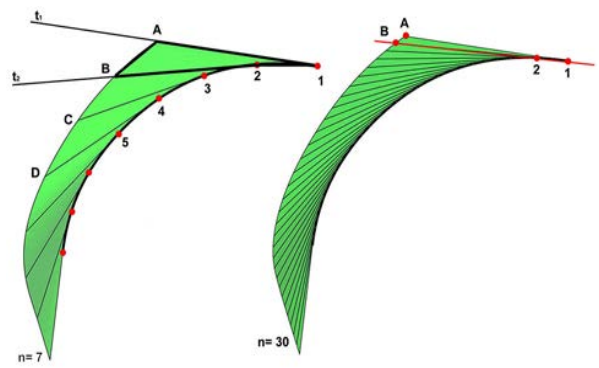

Figure 3: division of the surface in triangular elements. In an infinitesimal neighborhood, points 1,2 and B can be considered aligned and the two consecutive tangents will be incident in point

1. It follows that the face $A 12 B$ can be considered equivalent to the triangular face $\mathrm{A} 1 \mathrm{~B}$.

By comparing the metric values of $3 \mathrm{D}$ surface (the length of the edges and the area) with the unrolled solutions, we achieve these results: in the first case, the unrolled surface is larger than the real one, while in the second case it is smaller. Therefore, in order to exactly manufacture the final designed 3D shape, in the first case, pieces must be overlapped (bending), in the second case we have to cut (kerfing), and in both cases material must be flexible.

\section{RESULTS AND DISCUSSION}

\section{CASE STUDY: DESIGN A LAMP USING A TANGENTIAL SURFACE}

In order to apply researches, hypothesis and experiments described above, we designed a lamp prototype using tangential developable surfaces to manufacture its light diffuser. Our main goals are testing different digital fabrication techniques to solve problems linked to approximation between real shape and unrolled shape and testing the same discretization technique for opposite manufacturing techniques to create developable and foldable strips starting from a rigid material.

Showed prototypes are composed by open polyhedra (adjacent planar faces) manufacture using Kerf-bending subtractive technique (e.g. Hyparsphere and Hyparcube prototypes, material: cardboard/wood) and 3D printing additive technique (Loop lamp, material: PLA on fabric net) to discretize and manufacture shapes deriving from the intersection between developable surfaces (cones, cylinders) and non-developable surfaces (quadrics) transformed into developable strips (open polyhedra).

Both these approaches allow to transform and develop a complex shape in one or a few reverse bendable pieces. Moving from kerfing (kerfs along consecutive triangles edges) to paneling (adjacent printed triangular pieces) technique, we present a method to construct a developable strip composed by consecutive adjacent triangular faces identified by consecutive incident lines.

Therefore, these experiments allow to demonstrate versatility of the same computational approach (discretization technique) to achieve different results about shapes and materials. Furthermore, designed shapes are also the result of an experimentation on the shadows created on a planar surface by the surface's own shape and placement relative to a light source (cast shadows).

The curves that we used to generate these tangential surfaces are the intersection between a circular cylinder, a circular cone or a sphere and an hyperbolic paraboloid, thus the projection of this curve on a plane, cast shadow, could be a circle in some particular cases. We studied the position of the light source in relation to space line and the plane where cast shadow goes.

First, to extract consecutive tangent lines we need to achieve a suitable regression edge working on the intersection between different shapes (we look for a functional and aesthetic curved shape).

Combination and intersection of different analytic surfaces allows to extract infinite developable shapes. Furthermore, a limit-shape (a cube, a sphere, a cone and so on) is necessary to cut tangent lines and to outline the outer edge to aesthetically and functionally complete the final prototypes.

Moreover, thanks this specific approach, we can divide and approximate $3 \mathrm{D}$ surfaces to cover a complex shape by developable strips. The geometric construction of the tangential surface used for the diffuser element of the lamp starts from the construction of the internal curve, determined in previous step by the intersection of a cylinder and a hyperbolic paraboloid.

According to previous premises, dividing the intersection curve (regression edge) in $n$ parts, our algorithmic definition allows to generate a ruled surface by constructing $n$ tangent lines (generating lines).

\section{SHAPING VS MANUFACTURING: TESTING}

The dissemination of digital fabrication techniques, subtractive and additive techniques, are introducing substantial changes in design. 
They are promoting the spread of manufacturing techniques that allow to manufacture both developable surfaces and double-curved surfaces.

To manufacture a developable surface, we need a flexible but not deformable material, we can make flexible a rigid material, or we can work to find special joints solutions.

To manufacture a tangential developable surface, first we tested kerf-bending technique, then we searched a 3D printed solution to achieve the same result.

\section{SUBTRACTIVE MANUFACTURING}

Kerf-bending (subtractive digital fabrication technique) allow to move from a flat element to a cold bent object according to a specific kerf-pattern. Kerf-bending technique consists in subtracting material in some points of a rigid panel or support to improve its flexibility.

About this topic, we are studying slit geometries and their pattern layouts, identifying two different approaches: uniform distribution or optimized distribution according to the curvature of surfaces and designed shapes (Güzelci et al., 2016; Muñoz et al., 2011).

The research for optimized solutions starts from knowledge of geometric nature of surfaces, particularly according to their structural curves networks (generating lines, principal curvature lines, isocurves, etc.). In our research we have tested different techniques to put staggered slits on developed surfaces, along their generating lines.

Therefore, our tested approach consists into a couple of main steps:

\section{1. development or flattening designed curved surfaces/shapes;}

\section{2. extraction of curves on which to distribute kerfing pattern.}

To manufacture a ruled shape according to a subtractive technique it is necessary to consider a pattern composed by staggered straight slits along developed generating lines of the surface. The overlapping of slits allows to cold bend a rigid material according to designed ruled shape. Dividing the regression edge into $n$ parts, our algorithmic definition allows to generate infinite tangent lines.

Therefore, it is possible to construct a loft surface using the $n$ consecutive tangent lines as section curves (generating lines). This method allows to extract infinite developable surfaces by modifying regression edge and surfaces using to delimitate the final shape of the object. Extending tangent lines near the regression edge it is possible to identify the intersection points of consecutive crossed tangents pairs. At the same time, it is possible to consider the intersections points between consecutive tangents and delimiting cube or sphere (Figures 4 and 5).

According to elementary geometry, we know that crossed straight lines and a triad of not aligned points identify a plane. Likewise, these points joined to intersection points between tangents lines and cube (or sphere) represent the vertices of consecutive triangular faces that make up the polyhedral strip. Unroll component (Lunchbox, Grasshopper add-on, Rhino3D_McNeel), allows to automatically develop this polyhedral strip composed by planar triangular faces.

Therefore, thanks this specific approach, we can divide and approximate 3D surfaces to cover a complex shape by developable strips, which can be unfolded to the plane without stretching or tearing. Figure 5 shows the comparison between Gaussian curvature analysis of polyhedral strip and generic patch surface: polyhedral strip is completely developable (green: $k=0$ ), while the generic patch presents mixed curvature.
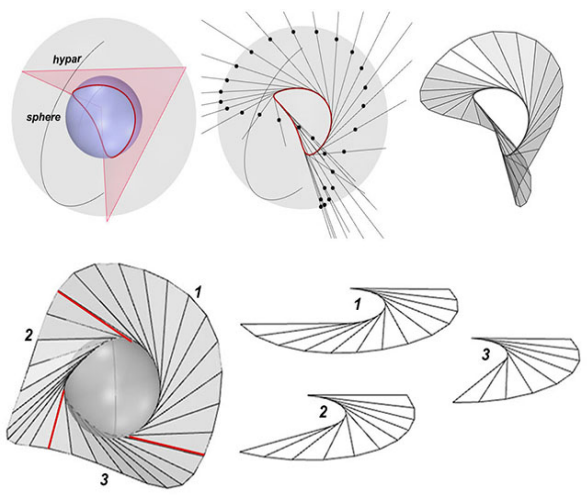

Figure 4: combination and intersection of different analytic surfaces allows to extract infinite developable tangentials.

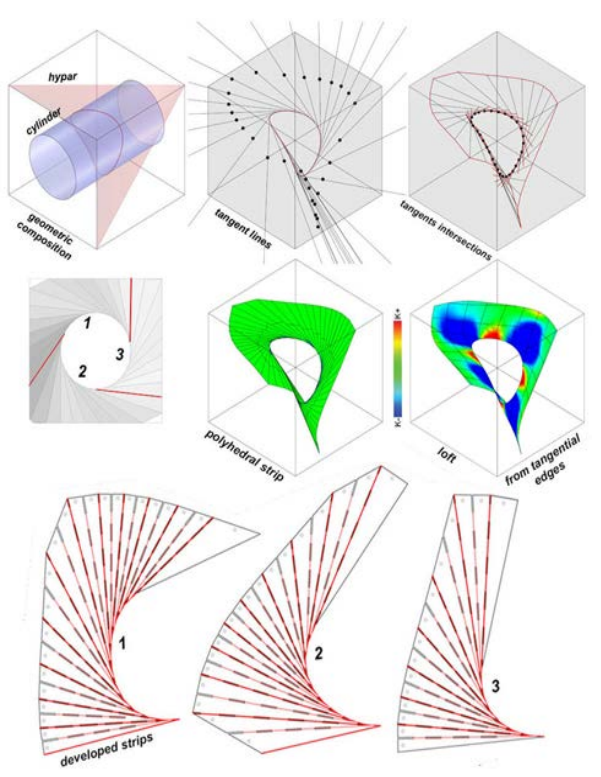

Figure 5: Tangential surfaces from shaping to manufacturing (subtractive digital fabrication techniques). Distribution of straight slits on generating lines of tangential surfaces unrolled pieces.

According to this process, we can fabricate generic 3D complex shapes cold bending several materials. We consider developable strip as a patch surface approximation and as a developable composition that best fits a non - developable strip.

To design complex surfaces can be economically expensive because manufacturing, assembly and maintenance of curved surfaces quite often is expensive. Moving from shaping to digital fabrication, we have distributed the kerf - pattern along the unrolled generating lines. We have manufactured the final piece dividing the entire strip into three different strips. Finally, we have divided all the generating lines in segments and we alternately choose and offset them to distribute staggered slits. Polyhedral strips division allows to avoid overlapping. Joining and fixing developed pieces each other, it is possible to observe that final shape cannot be flattened. 

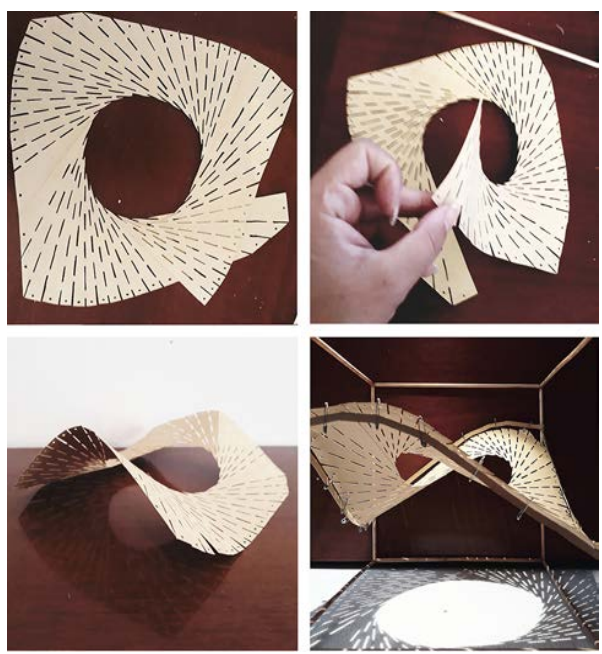

Figure 6: Tangential prototype (cardboard) manufactured according subtractive technique (Kerfing). Despite material, the whole final curved shape, composed by three fixed polyhedral strips (fig. 5), takes on designed shape and it cannot be flattened.

Therefore, it is necessary to create a system of buttons or hooks to join and disconnect the adjacent edges of the unrolled strips. Figure 6 shows shadows effects and geometries resulting from this composition.

Digital experiments to improve slits distribution on generic tangential surface are in progress. More precisely, we are testing algorithmic tools to parametrize patterns on curved and planar surfaces according to their curvature and correspondent developed configuration. We extract from flatten profiles a $2 \mathrm{D}$ grid and we vary the number of consecutive generating lines to modify grid step. Then, to generate an optimized kerf-pattern on flatten profile, it is possible to stretches a matrix into the polygonal frames that compose the regular or irregular grid (regularity depends on the surface genesis according to the distribution of the generating lines). We already reach results for cylindrical, conical and helical shapes.

\section{ADDITIVE MANUFACTURING}

To manufacture the light diffuser (developable tangential surface) of the lamp according to an additive technique, we tested 3D printing of PLA on fabrics to achieve a composed flexible material. Then, we choosed some fabric samples, selecting different materials according to their weave width and elasticity: jersey, cotton sheet, metal and plastic mosquito net and tulle (Figure 7). Testing different patterns, the best way to achieve a tangential surface is to manufacture separated triangular faces built on the generating lines of developable ruled surface. Generic tessellation on fabric (in this case triangulation) allows great flexibility but it does not allow to correctly follow the designed shape (Figure 8). The correspondence between digital and real prototype is important to achieve designed shape and shadow effects.

These considerations validates the passage from kerfing to paneling techniques according to the same shaping and discretization technique and it validates tangential strips as the geometric solution that best fits an open generic curved strip to faithfully manufacture a specific designed element (Lanzara, 2019, pp. 183 - 185), (Figure 9).
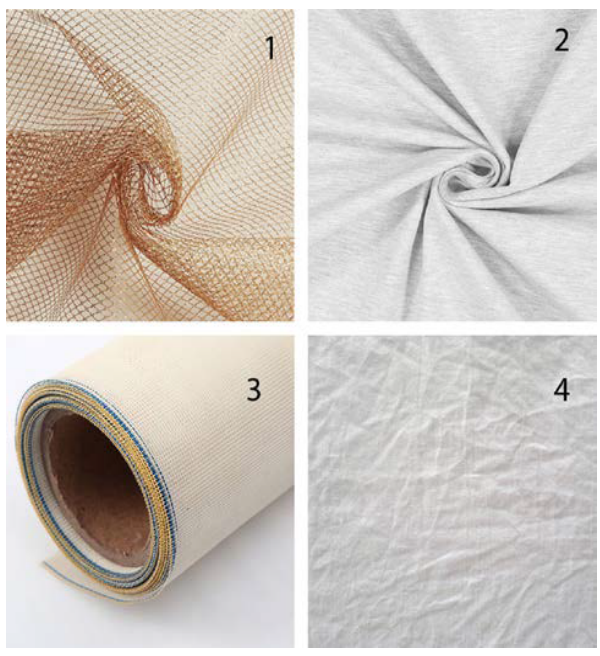

Figure 7: Tested fabric samples (3) according to weave width and elasticity: metal and plastic mosquito net (1), jersey, cotton sheet (2) and tulle (4).
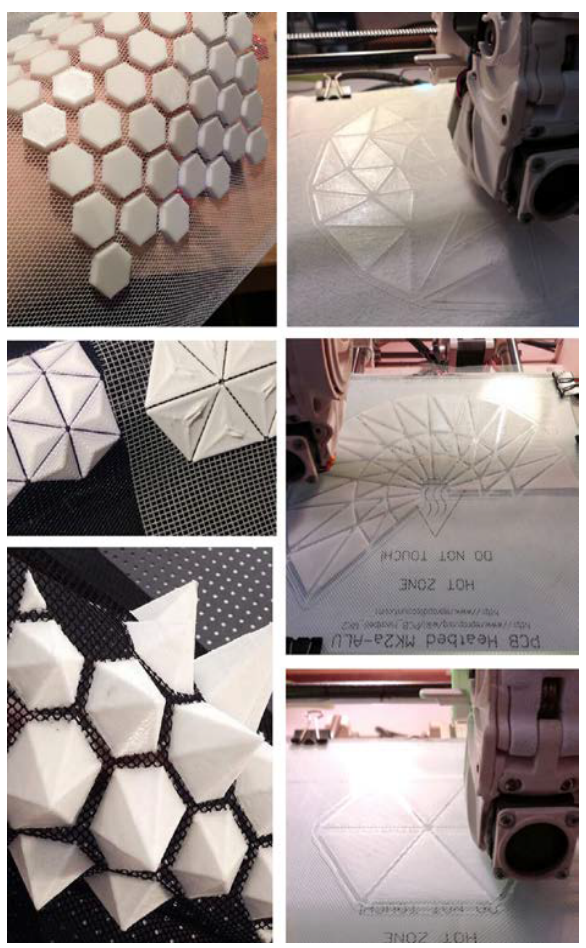

Figure 8: Testing of 3D printing triangulated pattern on fabrics. 

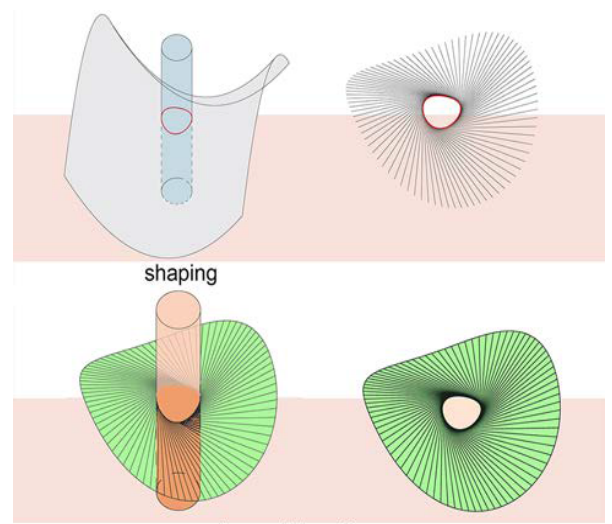

tangential surface

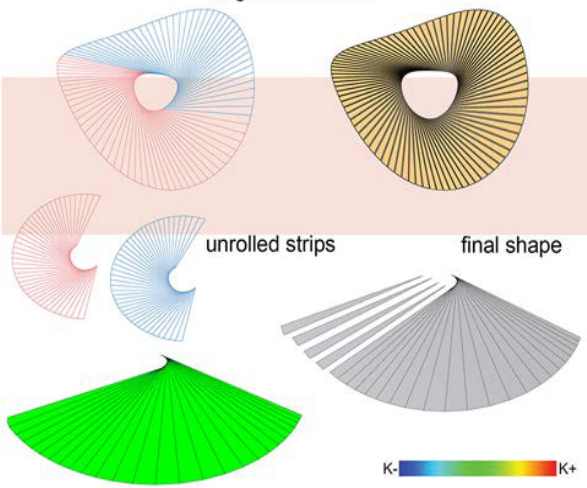

Figure 9: Shaping of 3D printed lamp light diffuser. The regression edge derives from the intersection between a cylinder and a hyperbolic paraboloid. The border shape derives from the intersection between the tangential surface and a cone. Tangential surface is developed as a set of separated triangles.

Thermofused plastic needs to penetrate inside the material merging with it to achieve an hybrid composed material able to faithfully follow designed pieces. All the attempts on mosquito plastic and metal net were unsuccessful for the difficulty in uniformly stretch and fix the material on the printer's plate. Therefore, tulle represent the best solution to manufacture the shape thanks to its light, flexibility (to be stretched uniformly on $3 \mathrm{D}$ printer plate) and texture that allows the heat-melted plastic material to penetrate and adhere to it (Figure 10).

To compose diffuser, it is necessary to separately print surface borders to generate a frame composed by two curved elements. Finally, we need to define the spaces (thickness $1 \mathrm{~mm}$ ) between triangular developed faces to bend the diffuser (exactly following its designed shape), and to achieve light and shadow effects deriving by the whole shape and the relationship between full and empty spaces. (Figures 11 - 12).

The upper element that shields and directs the light from the bulb is conceived as a cone, whose curved shape is determined by extruding from a point (the vertex of the cone) the diffuser profile, to give coherence between the parts. Although detached and of different sizes, all the pieces assume uniformity because of their geometric formal coherence (Figures 13 - 14).

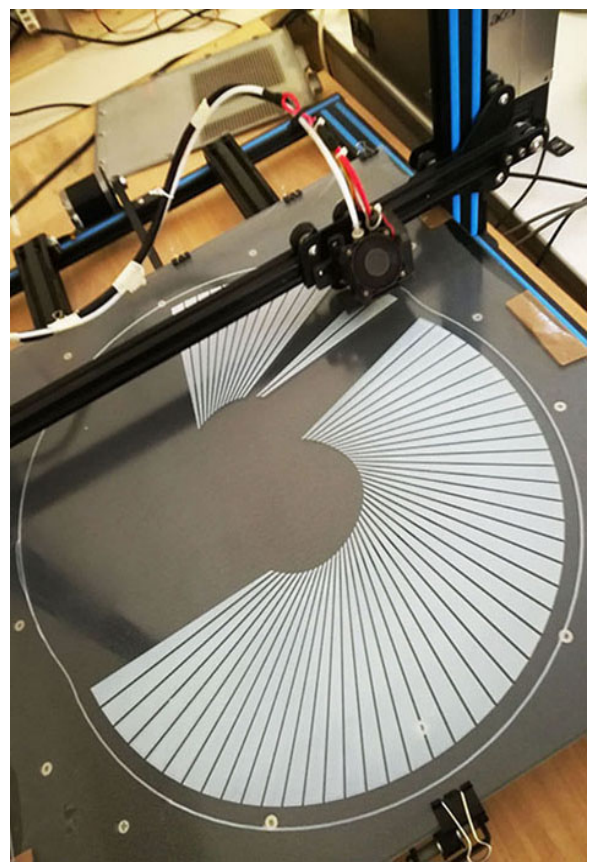

Figure 10: Tangential prototype manufactured according additive technique (paneling - PLA 3D printing on tulle). Tulle allows the heat-melted plastic material to penetrate and adhere to the fabric.
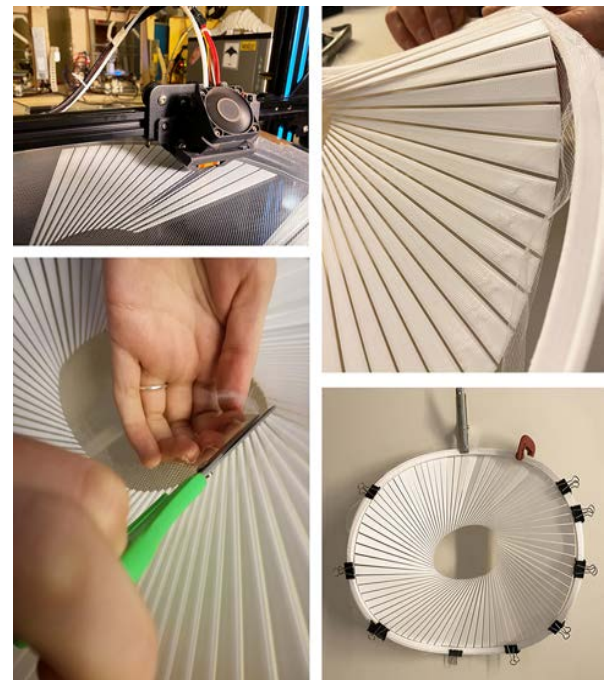

Figure 11: Curved borders allow to fix tulle

to put in shape the couple of unrolled pieces that compose the whole light diffuser. 

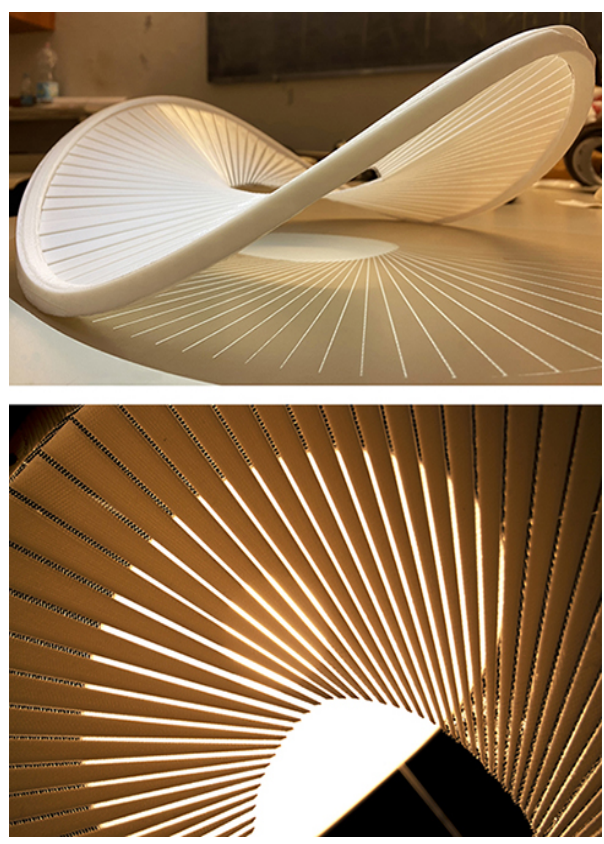

Figure 12: 3D printed lamp diffuser: surface borders generate a frame to fix composite solution (tulle and PLA)

Slits allow to bend the shape and to achieve designed light and shadow effects.

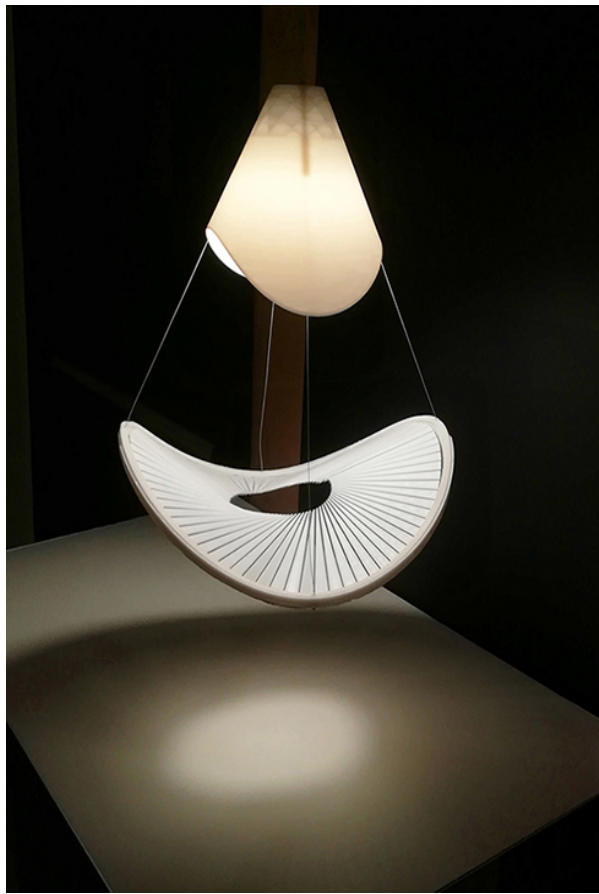

Figure 13: Loop lamp. Final prototype is the result of an experimentation of shadows on planar surfaces.

The upper element is conceived as a cone as extrusion of diffuser profile.

\section{CONCLUSION}

According to these results, our testing activity is aimed at verifying combination between a digital discretization technique and different manufacturing techniques to create developable and foldable strips starting from rigid materials. This approach allows to transform and develop a complex shape in one or a few reverse bendable pieces. Therefore, these experiments allow to demonstrate and validate versatility of the same computational approach to achieve different results about combination of shapes and materials.

Future works may consider these approaches to design and manufacture other complex geometric configurations testing the same techniques on existent or new materials. A possible advance is to test kerfing also on PLA, plastics or other composite materials. Furthermore, the same process could be considered to approach multiscale design, discretization and manufacturing of complex surfaces, working on the decomposition of whole shapes and manipulating single pieces to optimize their design and digital manufacturing.

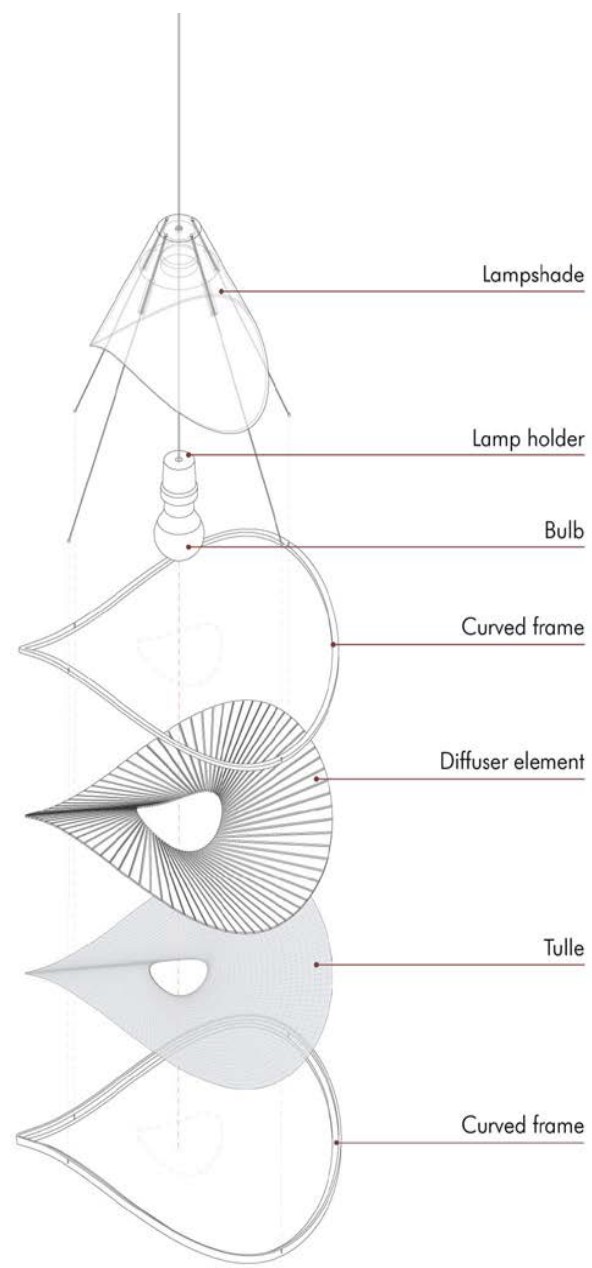

Figure 14: Loop lamp composition. All parts are collected by a conical shape. 


\section{REFERENCES}

Capone, M. (2018). Teorie e metodi per lo sviluppo delle rigate e lo spianamento approssimato delle rigate non sviluppabili. Disegno, 3, $53-68$.

Capone, M., \& Lanzara, E. (2018). Kerf-bending: ruled double curved surfaces manufacturing. In TECHNOPOLITICAS. SIGraDi, XXII Congreso da Sociedade Iberoamericana de Gráfica Digital, Universidade de São Paulo, São Carlos, Brazil. 7-9 November, 2018, pp. 653 - 660. Sao Paulo: Blucher.

Capone, M., \& Lanzara, E. (2019). Parametric Kerf-bending. Manufacturing a double curvature surfaces for wooden furniture design. In F. Bianconi, \& M. Filippucci (Eds.), Digital Wood Design. Innovative techniques of representation in architectural design, (pp. 415 - 439). Springer International Publishing.

Fallavollita, F. (2009). Le superfici rigate. In R. Migliari, Geometria Descrittiva. Vol. 2. Tecniche e Applicazioni, (pp. 153 - 223). Novara: CittaStudi Edizioni.

Farinella, C., \& Baglioni, L. (2017). Modello come immagine mentale. La diffusione dei modelli matematici nell'arte d'avanguardia e la superficie sviluppabile di Antoine Pevsner. In Territori e frontiere della Rappresentazione/Territories and frontiers of Representation, (pp. 929 - 936). Roma: Gangemi Editore.
Güzelci, O., Bacınoğlu, Z., \& Alaçam, S. (2016). Enhancing flexibility of 2D planar materials by applying cut patterns for hands on study models. In SIGraDi, XX Congreso de la Sociedad Iberoamericana de GráficaDigital, Buenos Aires, Argentina. 9-11 November, 2016

Lanzara, E. (2019). Shaping\&Paneling. Superfici complesse per l'architettura e il design. Milano: FrancoAngeli,

Lawrence, S., (2011). Developable Surfaces: Their History and Application. Nexus Network Journal, 13, p. 701.

Menges A. (2011). Integrative Design Computation integrating material behavior and robotic manufacturing processes in computational design for performative wood constructions. In Joshua M. Taron, Integration Through Computation. Proceedings of the $31^{\text {st }}$ Annual Conference of the Association for Computer Aided Design in Architecture (ACADIA), pp. 72-81. Banff (Canada). 13-16 October 2011.

Migliari, R. (2009). Geometria Descrittiva. Vol. 2. Tecniche e Applicazioni. Novara: CittaStudi Edizioni.

Muñoz, P., Coronel, J. L., Sequeira, A., \& Magnasco, I. R. (2011) Fabricación digital y morfología: la flexibilidad en la generacion de la forma. Ediciones de la forma. In SIGraDI, XV Congreso de la Sociedad Iberoamericana de Gráfica Digital. Santa Fe, Argentina. 23-25 November, 2011. 